\title{
Flexible Cooperative Game Theory Tool for Peer-to-Peer Energy Trading Analysis
}

\author{
Julian Dixon*, Malcolm McCulloch ${ }^{\dagger}$, Thomas Morstyn ${ }^{\ddagger}$ and Liyang $\operatorname{Han}^{\S}$ \\ Department of Engineering Science, University of Oxford \\ Oxford, UK \\ Email: *jmdixon100@googlemail.com, $\left\{{ }^{\dagger}\right.$ malcolm.mcculloch, ${ }^{\ddagger}$ thomas.morstyn, ${ }^{\S}$ liyang.han $\} @$ eng.ox.ac.uk
}

\begin{abstract}
This paper presents an extensible and adaptable tool with which to study the use of cooperative game theory for allocating rewards to prosumers in peer-to-peer energy trading systems. With the aim of expanding the use of renewable generation, the tool is a combination of the cooperative game theory platform and a specific game which focuses on a prosumer peer-to-peer (P2P) network in a distribution network feeder. The final platform is able to run user defined P2P trading games, including the aforementioned one, over multiple trading intervals. It has two inbuilt allocation schemes which can be tested on the games for fairness and stability.
\end{abstract}

Index Terms-Cooperative Game Theory - Distributed Networks - Shapley Value - nucleolus

\section{INTRODUCTION}

The transition towards renewable energy generation will bring a fundamental change in the structure of the energy distribution grid, from a vertical energy model towards a horizontal one. This essentially means that individuals will be capable of both consuming and producing energy ('prosuming') without the need for centralised power plants [1]. The intermittent and heterogeneous nature of prosumer resources means that prosumers can benefit from $\mathrm{P} 2 \mathrm{P}$ energy trading and that the grid will have to adapt to accommodate for this new distribution model [2].

Here, we investigate prosumers' reactions to different $\mathrm{P} 2 \mathrm{P}$ schemes and in particular, what would incentivise them to participate. One of the aspects is to look at how game theory can aid the fair distribution of profit in a renewable based $\mathrm{P} 2 \mathrm{P}$ scheme. As cooperative game theory allows a reward allocation method to be set up to apply to various player behavioural scenarios, a single platform can run multiple unique $\mathrm{P} 2 \mathrm{P}$ trading games.

In comparison to competitive game theory, where players act to maximise benefit for themselves [3], a cooperative game is one where the primary actions of the group maximise the overall benefit to the group [4]. Only once this course of action has been taken, are the rewards for taking such actions divided out. This approach has already been applied to similar power system problems, such as aggregation of wind power [5] and electric vehicle charging [6].

This paper proposes a cooperative game theory energy trading platform. The first game to be developed: 'energy trading in a small distribution network', allows us to study the effects on individual participants of $\mathrm{P} 2 \mathrm{P}$ collaboration. The game also helps us understand the trade-offs of each allocation scheme for calculating the rewards given to the players, with regards to stability and inclusion in the trading group. The platform is designed in an extensible manner, allowing the possibility for it to be applied to other cooperative power system games.

The major contribution of this work is the establishment of a P2P cooperative game platform which can be configured to reflect various energy trading schemes, and to compare different reward allocation methods.

The rest of this paper is organised as follows: Section II presents an introduction to $\mathrm{P} 2 \mathrm{P}$ networks and section III to cooperative game theory. Section IV details the workings of the cooperative trading platform, whilst section $\mathrm{V}$ describes the first game that has been written to run on it. With these laid out, section VI explores different trading scenarios using the game and section VII discusses the wider implications of our findings for the P2P energy trading model.

\section{Peer-to-Peer Energy Trading Networks}

For the purpose of the game, we have chosen to use a 'distribution network feeder with residential PV generation and energy storage', which has been selected as this is an important problem class for studying the integration of distributed energy resources. This model has been selected as it allows us make use of the existing energy grid as a resource for energy security, permitting us to ignore the need for built in redundancy. A connection to the national grid also provides a good benchmark for the pricing structure of the system. In addition to the points outlined above, connectivity with the existing infrastructure means that we assume an $\mathrm{AC}$ distribution.

In general, electricity suppliers sell energy to consumers for a higher price than they offer for excess generation as inflexible generation is less valuable. If prosumers are able to cooperatively share energy and schedule their batteries, and then only buy/sell the group's net demand from their supplier, they can obtain more value from their distributed resources and a profit which needs to be allocated.

Profit should be allocated to any player who helps to balance the peer-to-peer system, either by a) storing energy so that it can be used when demand outstrips supply, or b) shifting their supply or demand so that it acts to balance the P2P network. We achieve this by charging the net energy surplus/deficit of 
any coalition at the respective market rate to calculate $v(C)$, and thus we ascertain the marginal stability any given player provides.

For the purpose of creating the game, the following assumptions have been made:

Assumption 1 (Half-hourly Meters). All energy generation and production usage information is received on a half hourly basis, the current standard for smart meters in building.

Assumption 2 (Perfect Information). All energy use and energy generation information is perfect. This simplification is so that the cooperative game theory can be tested in a 'perfect world' scenario.

Assumption 3 (Unconstrained Local Grid). The game further assumes a real power transfer model for the distribution network. This model does not consider reactive power flows, distribution network losses and power flow/voltage constraints and is commonly used for energy management in distribution networks [7].

Assumption 4 (Fixed Retail Supply Price). We assume that the external retail supplier does not adjust prices based on the actions of prosumers in the $\mathrm{P} 2 \mathrm{P}$ scheme.

\section{CoOperative GAMe Theory}

Here, we deal with the question of how to divided generated profit in a manner which incentivises investment and participation in the P2P scheme. The following sections will examine certain elements associated with cooperative games and how they can help us achieve our aims.

We will be dealing with a given set, $A$ of $n$ players which will be divided into coalitions, $C$.

\section{A. Coalitions and Stability}

Definition 1 (Coalition Structure). Our given set, $A$, is partitioned into subsets (coalitions), where each player is only a member of one subset. This structure $C S=\left[C_{1} \ldots C_{m}\right]$ satisfies two conditions: $U_{i=1}^{m} C_{i}=A$ and $i \neq j \Longrightarrow C_{i} \cap C_{j}=\emptyset$.

The value (worth) of a coalition can be calculated at any time-step during the game and is expressed by the characteristic function, $v$ thus; $v\left(C_{i}\right)$. An optimal coalition is one that maximises $v \sum_{i=1}^{m}\left(C_{i}\right)$. The marginal contribution of any player, $a$ to a coalition is the difference created in $C$ 's value by adding $a$ to it. As the set of imputations $I(v)$, with $v$ the characteristic function, is the set of allocations for the Grand Coalition (GC, the set $A$ ), the terms 'imputations' and 'allocations' will henceforth be used interchangeably.

\section{B. Convex Games and the Core}

Definition 2 (Convexity [8]). In a convex game, the incentive for joining a coalition grows with the size of the coalition: $v\left(C_{1} \cup C_{2}\right)+v\left(C_{1} \cap C_{2}\right) \geqslant v\left(C_{1}\right)+v\left(C_{2}\right) \quad \forall C_{1}, C_{2} \subseteq A$. It follows that: $v\left(C_{1} \cup\{i\}-v\left(C_{1}\right)\right) \leqslant v\left(C_{2} \cup\{i\}-\right.$ $\left.v\left(C_{2}\right)\right) \quad \forall C_{1} \subseteq C_{2} \subseteq N \backslash\{i\}$ where $\{\mathrm{i}\}$ is an individual player.
The above result is of great significance as, if we can design our cooperative game to be convex, players will receive a greater reward for joining the GC as opposed to another sub coalition. As a result we can create a stable $\mathrm{P} 2 \mathrm{P}$ trading group [9].

The set of stabilising pay-off allocations for a coalition game with transferable pay-offs is called the 'core'. A transferable pay-off is where the reward can be transferred without loss to another player.

Definition 3 (The Core [10]). A pay-off distribution, $x$ (set of allocations) as a whole is said to be in the core when no set of players can achieve more value by forming a different coalition. Formally: $C(A, v)=\left\{x \mid \sum_{i-1}^{n} x_{i}=v(A) \forall C \subseteq\right.$ $\left.A \sum_{i-1}^{n} x_{i} \geqslant v(C)\right\}$

The core can be calculated from the excesses of each coalition. For coalition $C_{1}$ : excess, $e\left(x, C_{1}\right)=v\left(C_{1}\right)-\sum_{j \in C_{1}} x_{j}$. The imputation is then found to be in the core if $e(x, C) \leqslant$ $0, \forall C$, in other words if there is no excess value in the pay-off distribution. Crucially this means that all sets of imputations in the core exhibit the property of group rationality.

However, as there can be multiple imputations in the core, finding a set of allocations in the core does not unequivocally prove convexity.

\section{Shapley Value}

Having detailed theoretical attributes of cooperative game theory in sections III-A through III-B, this section will now explore one possible solution to creating a 'sensible' pay-off vector. Introduced in [11], the principle of the Shapley Value is that it assigns each player their expected marginal contribution to the value of the $\mathrm{GC}$, assuming the players join in random order. The sum is weighted to take into account the different joining orders with which each coalition could be formed. The value is calculated as:

$$
\phi_{i}(v)=\sum_{C \subseteq N \backslash\{i\}} \frac{|C| !(n-|C|-1) !}{n !}(v(C \cup\{i\})-v(C))
$$
$11]$

The Shapley value is based upon the following axioms, which ensure the fairness of the allocation:

- Symmetry: For two equivalent players, $i, j \in A$ in the sense that $v(C \cup\{i\})=v(C \cup\{j\})$, for every subset $C$ of $A$ which contains neither $i$ nor $j, \quad \phi_{i}(v)=\phi_{j}(v)$.

- Efficiency: The total profit is distributed amongst the players: $v(A)=\sum_{i=1}^{n} \phi_{i}$ where $\phi_{i}$ are the individual allocations to the players.

- Additivity: For two games, described by value functions $v$ and $w: \phi(v)+\phi(w)=\phi(v+w)$

From these follows the important dummy player property: if a player does not contribute to the value of any coalitions, 
their pay-off is 0. One major advantage of using the Shapley value is that it has a unique solution so that, even though the core may contain multiple pay-off distributions, calculating the Shapley value will only leave one pay-off option [12].

However, the Shapley Value imputation may not be in the core, if the game with which it is calculated is non-convex. In this instance players will not want to always be in the GC, destabilising the P2P network.

\section{D. nucleolus}

An alternative method to calculate the set of imputations is the nucleolus, which is realised by finding the most dissatisfied coalition with a proposed initial imputation and minimising this worst inequity. This process is repeated until the excess vector is minimised lexicographically.

If the nucleolus is in the core then the game is said to be 'balanced' [13]. In one respect, a balanced game is still sufficient for our P2P system as it ensures, by using the nucleolus as the pay-off distribution, no player is better off outside the GC. On the other hand, it does not have a closed form of expression and often precludes the 'fairness' axioms upon which the Shapley value is based.

\section{Cooperative Game Theory Tool}

The 'Cooperative Trading Platform' (CTP) was written in Python, a dynamic strongly-typed language that is suitable for a modular and extensible program. An overall UML of the implementation is shown in figure 1. The tool works as follows:

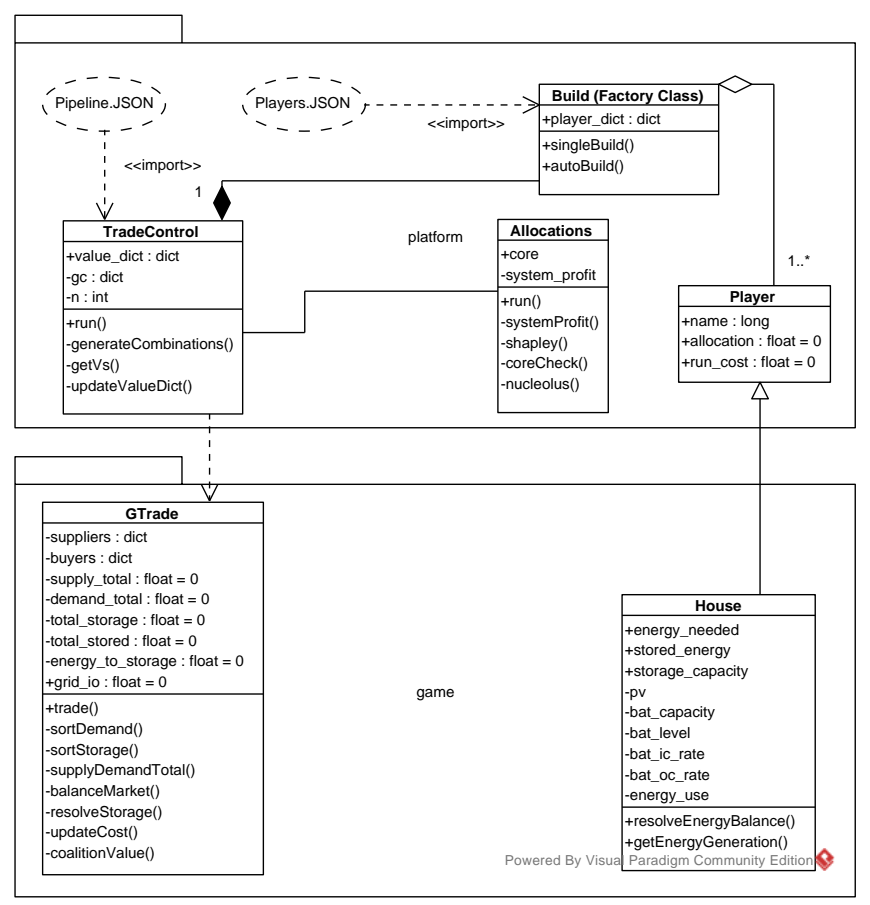

Fig. 1. Class UML for the program, not showing output and auxiliary classes.
1) Individual players are built as instances from any number of different player (object) types found in the 'Game' directory.

2) The players are passed to 'TradeControl' (TC) which implements the cooperative game theory aspect of the program. Here all of the possible combinations of the players are formed and passed to the specific game for a set number of time-steps.

3) The specific game runs with the players and returns to TC a dictionary of the coalition values, $v(C)$, as described in section III, which are then stored as a variable of the TC instance associated with that time period of the run.

4) From this dictionary of coalition values, a pay-off vector can be calculated by the 'Allocations' class using either the Shapley Value or nucleolus allocation scheme.

5) Individual allocations from the pay-off vector are then passed back to the respective players.

6) From here, players can be queried for visual and statistical outputs.

In order to establish the effects of using cooperative game theory to allocate profit over a long time period the tool is then able to seed new coalitions using the final state of players from the GC of the previous game (as seen if figure 2). This $\mathrm{GC}$ seeding process is imperative as it prevents divergence of coalition states over the course of multiple games.

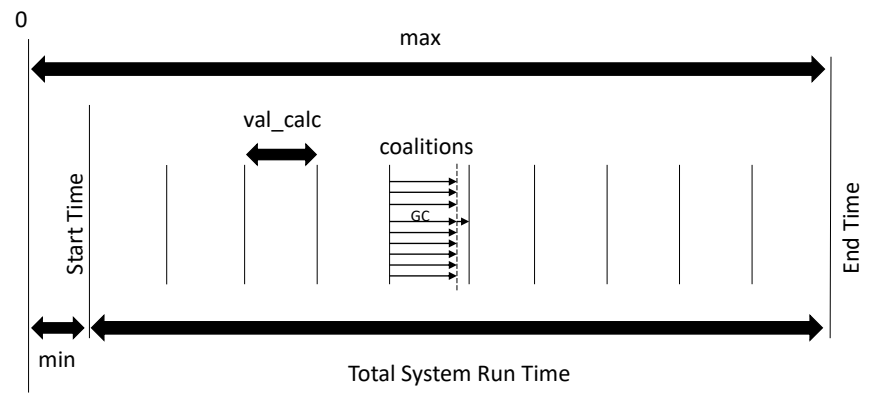

Fig. 2. Diagram detailing internal system timings for the platform, showing multiple games being played sequentially between the min and max input number of timesteps, each over a 'val_calc' number of steps

\section{A Peer to Peer Energy Trading Game}

The cooperative game simulates peer-to-peer energy trading in a small distribution network, using the 'connected microgrid' model, as shown in figure 3 . The model has deliberately been kept small, running in the region of 2-15 players each time, as the computational complexity of calculating the $v(C)$ for each coalition is $O(n ! /(n-k) ! k !)$, where $\mathrm{n}$ is the total number of players and $\mathrm{k}$ the number of players in the coalition, thus requiring the total number of players to be kept small for a suitable computation time. An overview of the game's design is shown in figure 4.

\section{A. Game Players}

Each player in the game has been set up to simulate a house in the distribution network, with it's own energy use, 


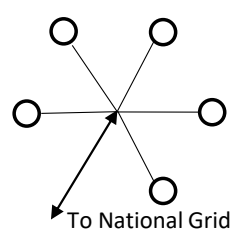

Fig. 3. Diagram of a distribution network feeder

generation and storage facilities. Real energy consumption data has been used from the London data store [14], which was collected as part of the UK Power Networks led 'Low Carbon London' project between the years 2011 and 2014.

Energy generation is from PV cells and is modelled by a truncated Gaussian distribution. Each house has a battery where the total energy capacity and the charge/discharge rates (C-rates) are input parameters. Storing energy over the end of a value calculation period will either falsely create or remove value from a coalition. Thus, at the end of every calculation period the stored energy in every coalition is worked into the $v(C)$ at the buying price of the retail market, which only works with fixed market prices.

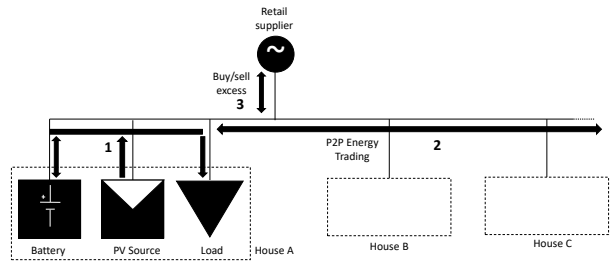

Fig. 4. Overview of the game with numbered steps 1) Houses try to balance themselves 2) The P2P scheme tries to balance itself 3) Surplus/deceit energy traded with the grid

\section{B. Energy Balancing}

At every time-step each house first tries to balance its own energy needs. It achieves this by subtracting the energy it consumes from that which it has produced and then using its battery to balance the difference. The battery is not only limited by either the amount of energy it has stored or its spare capacity, but also by its C-rate, the amount of energy it can source or sink over the given half hour period.

Once the house has tried to balance itself, any demand or surplus it is unable to cope with is sent as an 'energy needed' request (positive or negative) to the $\mathrm{P} 2 \mathrm{P}$ pool. At the same time, each house tells the pool the maximum amount of energy it can store from the pool and the maximum amount of energy its battery can give to the pool. This process is the nested loop in algorithm 1 and is much the same for the P2P system itself. However, in the situation where the retail price is time-varying algorithm 1 is not guaranteed to give the optimal solution, and a linear optimization would need to be used instead.

\section{The Role of the Energy Supplier}

As a consequence of assumption 4, we can use the retail prices to constrain the prices in the $\mathrm{P} 2 \mathrm{P}$ scheme. If each

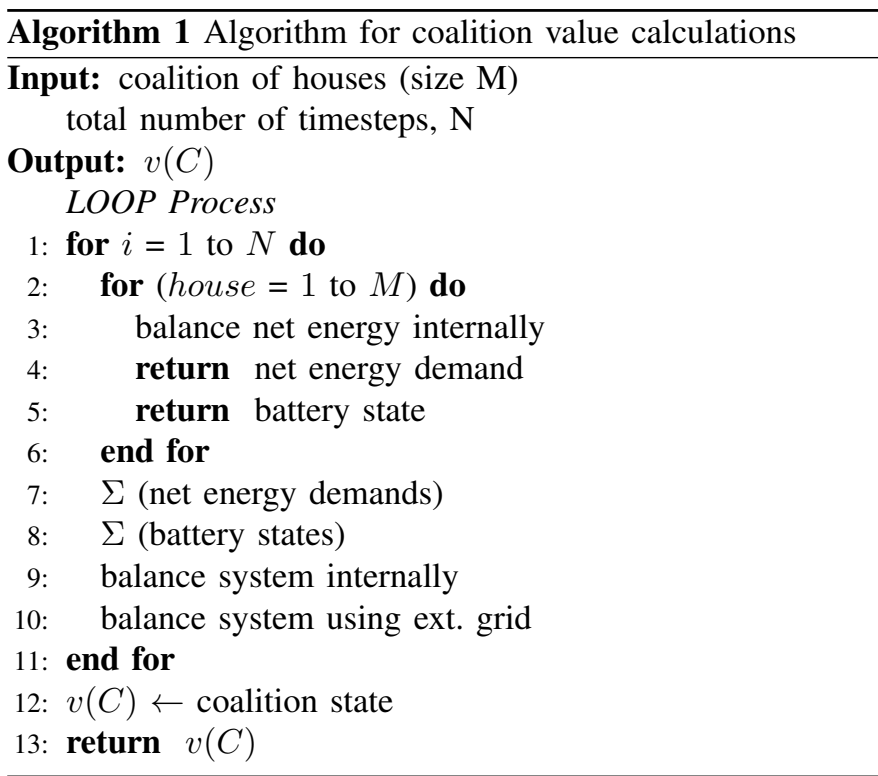

coalition is taken as a black box system and the net energy flow into or out of it is charged at the external retail price, we can determine the value created by the players in that coalition working together rather than separately. Thus the coalition's worth is $v(C)=P(C)-\sum_{i=1}^{N} P(\{i\})$, where $P$ denotes the profit that the load shifting creates, of either the coalition $P(C)$ or the players acting alone $(i=1: N)$, over the course of that value calculation period. An extension to the game would be to consider micro-grids which may continue operating when islanded.

\section{CASE STUdY}

This case study considers a direct comparison of the Shapley value and nucleolus allocation methods for four houses. Imputations from the nucleolus that are within the core only ensure that no player can achieve a better allocation than they receive in the GC, rather than the fairness properties guaranteed by the Shapley value. Thus, we specifically focus on determining which players are better and worse off under each different calculation regime.

\section{A. Inputs}

As seen in table I, houses A and B have large PV generation capacity, minimising the system's reliance on the national grid. Houses $\mathrm{A}, \mathrm{B}$ and $\mathrm{C}$ have batteries, with $\mathrm{C}$ only having a large storage capability and no generation itself. Houses A-C have an energy use profile which suggest no one is at home during the day, whilst house D has more constant energy use (figure $5 b)$. House D represents a house which have failed to invest in renewable technology, but have still signed up to be part of a P2P scheme.

The study runs the game for 400 half-hour timesteps (4 days), with pay-off allocations every 10 timesteps (5 hours). xternal Retail suppler sells energy to the prosumer coalition for $5 \mathrm{p} / \mathrm{KWh}$ and buys energy for $1.5 \mathrm{p} / \mathrm{KWh}$. 
TABLE I

INPUTS TO THE FOUR HOUSES RUNNING FOR THE CASE STUDY.

\begin{tabular}{lllll}
\hline House & A & B & C & D \\
\hline Battery Capacity $(k W h)$ & 13.4 & 7 & 20 & 0 \\
PV roof area $\left(m^{2}\right)$ & 120 & 80 & 0 & 0 \\
In/Out C-Rate $(k W h / 30$ minutes $)$ & 5 & 5 & 5 & 0 \\
\hline
\end{tabular}

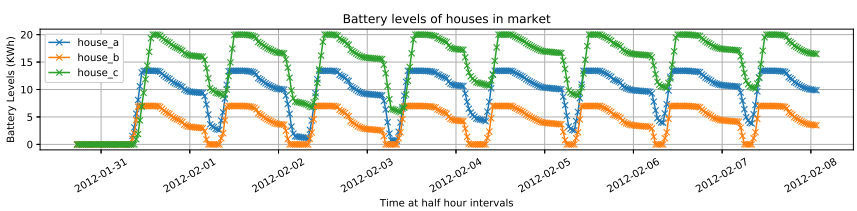

(a)

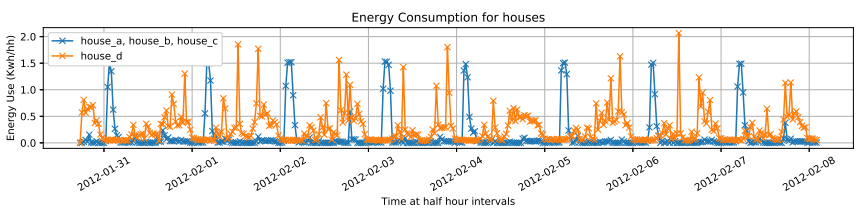

(b)

Fig. 5. House properties over time: (a) battery levels (b) energy use pattern

\section{B. Results}

Figure 6 shows the allocations rewarded to each house, in excess of what they would have paid the external supplier if they had not been in a P2P trading scheme, by (a) the Shapley value and (b) the nucleolus.

Each day house $\mathrm{C}$ is rewarded for storing the excess generation of houses $\mathrm{A}$ and $\mathrm{B}$, which do not have sufficient storage capacity of their own. As there is always sufficient energy in the system (ref. figure 5a), the allocations rewarded are never 0 . House $\mathrm{D}$ shows that, as a unique energy use profile benefits $\mathrm{P} 2 \mathrm{P}$ trading, it is still technically feasible to benefit a P2P scheme (and thus collect a reward) without making an investment. Despite house D having a large pay-off vector at the time of peak generation, a lack of generation or storage creates a volatile imputation.

The results of the Shapley allocation scheme are not always in the core and therefore it would benefit some of the houses to break from the GC and either trade on their in smaller groups or individually. As the nucleolus is always in the core and thus forms a stable GC it is the preferential allocation method, despite not possessing all the fairness axioms of the Shapley value. The most noticeable difference is that houses with increased storage benefit more under the set of nucleolus imputations than under the Shapley ones, at the expense of producers of renewable energy by PV panels.

\section{CONCLUSION}

This paper presented a tool with which to study the use of cooperative game theory for allocating rewards to prosumers in P2P systems. We have also investigated energy trading in a small distribution network, finding which imputation scheme

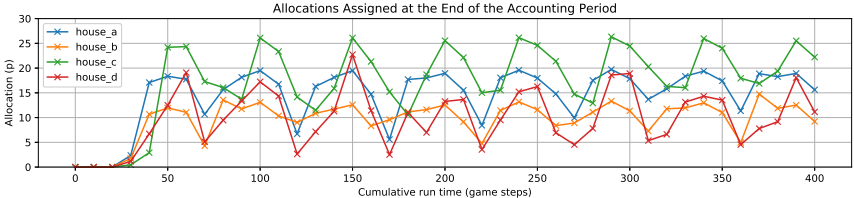

(a)

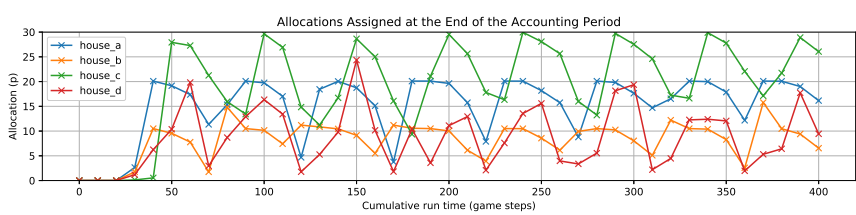

(b)

Fig. 6. Allocations to each house at the end of every game (value calculation) period (a) by the Shapley value (b) by the nucleolus

best enables us to incentivise investment and participation in such a P2P scheme. Further work could include tuning the tool to run with a larger pool of players or developing games with different rules.

A limitation is the computational complexity. This is an area for future research to investigate suitable approximation and sampling strategies, such as those proposed by Maleki [12]. A further interesting extension to the current game, would enable it to work with time varying external energy prices.

\section{REFERENCES}

[1] B. A. Dimeas, S. Drenkard, N. Hatziargyriou, S. Karnouskos, K. Kok, and J. Ringelstein, "Smart houses in the smart grid," IEEE Electrification Magazine, vol. March, pp. 81-93, 2014.

[2] Y. Parag and B. Sovacool, "Electricity market design for the prosumer era," Nature Energy, vol. 1, no. 4, pp. 1-6, 2016.

[3] M. Osborne and A. Rubenstein, A Course in Game Theory, 5th ed. MIT Press, 1998.

[4] J. von Neumann and O. Morgenstern, Theory of Games and Economic Behaviour. Princeton University Press, 1944.

[5] E. Baeyens, E. Y. Bitar, P. P. Khargonekar, and K. Poolla, "Coalitional aggregation of wind power," IEEE Transactions on Power Systems, vol. 28 , no. 4, pp. 3774-3784, 2013.

[6] R. Li, Q. Wu, and S. S. Oren, "Distribution locational marginal pricing for optimal electric vehicle charging management," IEEE Transactions on Power Systems, vol. 29, no. 1, pp. 203-211, 2014.

[7] T. Morstyn, B. Hredzak, and V. G. Agelidis, "Control strategies for microgrids with distributed energy storage systems: An overview," IEEE Trans. Smart Grid, 2016.

[8] L. S. Shapley, "Cores of convex games," International Journal of Game Theory, vol. 1, no. 1, pp. 11-26, 1971

[9] T. Dreissen, Cooperative Games, Solutions and Applications. Springer, 1988.

[10] D. Gillies, "Solutions to general non-zero-sum games," in Contributions to the Theory of Games IV, A. W. Tucker and R. D. Luce, Eds. Princeton University Press, 1959, pp. 47-85.

[11] L. S. Shapley, "A value for n person games," in The Shapley Value, A. Roth, Ed. Cambridge University Press, 1953.

[12] S. Maleki, "Addressing the computational issues of the shapley value with applications in the smart grid," Doctoral, University of Southampton, 2015

[13] D. Schmeidler, "The nucleolus of a characteristic function game," SIAM J. Appl. Maths, vol. 16, no. 6, pp. 1163-1170, 1969.

[14] L. Assembly. (2015) Smartmeter energy consumption data in london households. [Online]. Available: https://data.london.gov.uk/ dataset/smartmeter-energy-use-data-in-london-households 